\title{
UNA NECESIDAD CURRICULAR ANTE LOS PROCESOS HOMOGENEIZANTES DE LA EDUCACIÓN CONTEMPORÁNEA
}

\author{
M.Sc. Rafael Jiménez Corrales ${ }^{1}$
}

\section{RESUMEN}

El presente artículo trata de analizar la creación de las propuestas curriculares como producto de la convergencia de microprocesos institucionales y personales y macroprocesos sociales, lo cual se denomina en la teoría sociológica como desarrollos sintéticos. El escrito parte de la idea de que la creación de productos curriculares sólidos, transita por procesos históricos previos, los cuales son sistematizados e interpretados por los actores sociales en escenarios a veces antagónicos académicamente. Para ilustrar tales procesos, utiliza el ejemplo de la creación de la Maestría en Pedagogía con énfasis en Diversidad en los Procesos Educativos de la División de Educación Básica del Centro de Investigación y Docencia de la Universidad Nacional, donde enfatiza en el impacto que los cambios sociales van generando en la discusión académica y cómo esta va convergiendo en la creación de un plan de estudios y en el desarrollo de una carrera.

\section{Palabras claves}

Procesos educativos, diversidad, diseño curricular, Docencia y procesos sociales.

\section{Abstract}

This article is an attempt to analyze the creation of new curricular proposals as a product of the convergence of institutional and personal micro processes as well as social macro processes, which are known in sociological theory as synthetic developments. This article starts on the idea that the creation of solid curricular products departs from previous historical processes, which are then systematized and interpreted by the social actors involved, usually since opposite

1 Docente investigador de la División de Educación Básica, CIDE-UNA. Coordinador de la Maestría en Pedagogía con énfasis en Divcrsidad en los Procesos Educativos 2003-2006. Máster en Evaluación Académica y Licenciado en Historia. 
academic sceneries. In order to illustrate these processes, the author makes use of the construction of the Master's Degree Program in "Pedagogy of Diversity in Educational Processes" as an example, emphasizing the impact that social changes have on academic discussions as well as its influence on the curricular design and career development.

\section{Key words}

Curricular proposals, curriculum, synthetic development, Master Degree Program in Pedagogy of Diversity in the Educational Processes

Encuadre teórico para el análisis curricular a la luz de la creación de la Maestría en Pedagogía con énfasis en Diversidad en los Procesos Educativos

Comidis omprender la génesis de la Maestría en Pedagogía con énfasis en Diversidad en los Procesos Educativos, desarrollada en la División de Educación Básica (DEB) del Centro de Investigación y Docencia en Educación (CIDE) de la Universidad Nacional (UNA), no es tarea fácil, como tampoco lo es historiar procesos curriculares. La amplitud de las dimensiones que convergen en la creación de propuestas curriculares, es basta y profunda, quizá inagotable. Tales dimensiones implican desde los cambios sociohistóricos (a veces poco visibles en el momento) hasta ideas personales, historias cotidianas de los sujetos sociales. No obstante, como señala Posner (2000, p. 37), la dimensión histórica es una de las llaves para la comprensión y posibilidad de clarificación conceptual en materia curricular:

"Los currículos, al igual que las constituciones, los tratados y las leyes, deben ser entendidos en términos de sus contextos históricos. ¿Quiénes fueron sus arquitectos y cuáles los principios que los guiaron? ¿A qué situación educativa existente-incluyendo el currículo actual-o a qué conjunto de problemas estaba dirigido? ¿A qué presión social o política respondia? ¿Cuál fue el centro del esfuerzo del diseño curricular?”

Un diseño curricular es un producto social complejo; es un punto de convergencia entre macrotendencias sociales, económicas, políticas, filosóficas, entre otras y microprocesos institucionales y voluntades colectivas e individuales. Ahora bien, una de las grandes limitaciones en los análisis y evaluaciones curriculares es la carencia de documentación que explicite la 
génesis de los diseños curriculares. Si bien es cierto, que muchos modelos para diseñar propuestas curriculares obligan o comprometen a los diseñadores a responder explícitamente por las ideas iniciales que dieron origen a una propuesta curricular, son pocos los actores que se dedican luego a repensar esos procesos genéricos y a mirar en retrospectiva los contextos en que se generaron. Tales reflexiones son un aporte directo a los evaluadores curriculares, sobre todo a la hora de señalar modificaciones, o bien, a suprimir una propuesta curricular. Explicitar el proceso de diseño es un indicador de si sus respuestas son pertinentes, sobre todo dado lo dinámico de los cambios en el campo educativo.

Es importante señalar, que una de las máximas para la construcción conceptual y capacidad analítica de los historiadores en particular y de la mayoría de los cientistas sociales en general, radica en la objetivación de los procesos, que requiere de un alejamiento, el cual actúa como recurso metodológico fundamental. En el caso del análisis curricular con perspectiva histórica, se requiere muchas veces de la ayuda del tiempo, aliado que permite superar lo anecdótico y que genera la posibilidad de mirar las tendencias y los procesos, ante todo, la concatenación entre las microtendencias y los macroprocesos.

En este artículo, se intenta explicar la génesis de la creación de la Maestría en Pedagogía con énfasis en Diversidad en los Procesos Educativos, en el seno de la División de Educación Básica del Centro de Investigación y Docencia en Educación de la Universidad Nacional (en adelante, DEB-CIDE). Se persigue mostrar cómo el diseño curricular, en muchos casos concretos, va más allá del trabajo inmediato y técnico de los diseñadores curriculares. El artículo busca, mediante un ejemplo concreto, problematizar en torno a una serie de dimensiones que se conjugan para generar propuestas curriculares de manera que se supere la idea del currículo como un producto exógeno del contexto social e histórico donde se produce.

Se persigue superar la acción del sujeto individual y se trata de mirar un producto curricular como la convergencia de voluntades colectivas que responden a acciones cotidianas institucionales entremezcladas con macroprocesos sociales. De esa forma, se parte de la idea de que no existe una propuesta curricular que sea solamente producto de la inspiración o capricho de un sujeto o sujetos sociales. En esta reflexión, se mira al sujeto social como un espacio de convergencia de lo individual con lo colectivo, lo macro con lo micro, lo psíquico con lo social. Intentar deslindar o trazar fronteras en esos procesos de concreción curricular es una tarea no sólo difícil, sino quizá 
utópica, porque la mujer y el hombre son seres sociales e individuales, que actúan como unidades integradas. El ser humano es una intersección única de los procesos macro y microsociales, es producto y productor de ideas sociales, y el currículo en sus manifestaciones físicas, en este caso un plan de estudios de maestría, es una idea social. Una propuesta curricular es la convergencia entre vidas y procesos sociales; no es el producto de iluminaciones metafísicas de individuos aislados; ningún producto humano lo es. Por otra parte, la mayoría de propuestas curriculares nacidas de los escritorios de un grupo pequeño de expertos, tienden a tener poca duración, o bien, a no ejecutarse en la práctica tal y como se habían diseñado. Señala Posner (2000):

"Los curriculos son construidos por grupos de individuos confrontados con situaciones que demandan una acción de su parte. Un curriculo es parte de un diálogo permanente entre personas con diferentes creencias sobre la educación y sobre su compromiso hacia ella y, en especial, con diferentes creencias de lo que los docentes deben enseñar a hacer..." (p. 37).

Por otra parte, se insiste en la idea sobre la relación de los macroprocesos sociales y las perspectivas microsociales, proceso complejo que se puede visualizar en los diseños curriculares, pero que a su vez constituye uno de los grandes retos explicativos de toda construcción social; en este sentido, indica Ritzer (2000):

"Ese desarrollo consiste en un interés cada vez mayor por la cuestión del vínculo micro-macro... analizaremos el desarrollo paralelo que ha experimentado la teoría sociológica europea: el aumento del interés por la relación enire la acción y la estructura. Como veremos, existen importantes semejanzas y diferencias entre la literatura estadounidense sobre el vínculo micro-macro y la europea sobre acción y estructura. Las literaturas macro-micro y acción-estructura pueden considerarse en si desarrollos sintéticos y, por tanto, como parte del movimiento general hacia la sintesis teóricas que hemos analizado..." (p. 444).

Es importante comprender y explicar los procesos curriculares desde lo que Ritzer denomina desarrollos sintéticos, ya que dicho abordaje representa un instrumento teórico-metodológico de gran poder explicativo, el cual incluso abre la posibilidad de entender e incidir en la fase de ejecución de 
las propuestas curriculares que, por lo general, implica contradicciones con respecto a la fase del diseño curricular.

\section{Antecedentes teóricos-históricos de la génesis de la Maestría-la profunda discusión académica}

Desde los albores de los años noventa, un sector de las académicas y los académicos de la DEB-CIDE, iniciaron un debate en torno a preguntas claves del quehacer educativo y sus implicaciones en la formación de docentes; tales preguntas pueden verse ahora clarificadas con los aportes de autores como Florez (1999), Florez y Tobón (2001) y Posner (2000), quienes sintetizan en lo que han denominado Modelo Pedagógico; al respecto, señala Florez,

"Todos los técnicos en diseño curricular reconocerian que sin metas de formación, sin claridad sobre cómo se jalona o se estimula el aprendiza$j e, y$ sin opciones razonadas sobre el tipo de experiencia o de contenidos que faciliten y aseguren cierta ganancia educativa en los alumnos, no seria posible formular un buen plan curricular. Pues bien, tales requerimientos no son más que los criterios de elegibilidad, o parámetros, que definen un modelo pedagógico" (Posner, 2000, p. XXVIII).

Dentro de las premisas básicas de la reflexión de ese grupo de académicas y académicas, se lograron clarificar dos:

1. La educación es una clara acción política, no existe educación neutral.

2. La formación docente debe optar por la conformación de una visión social y política en la acción docente de las y los estudiantes.

Obviamente, en dicho momento la pléyade de autores que parten de tal premisa no era tan extensa como lo es en la actualidad, sobre todo con la consolidación del movimiento de Pedagogía Crítica en los Estados Unidos. El autor que en ese momento había sostenido tal idea en América Latina era Paulo Freire. En la actualidad, los pedagogos que parten de esa premisa son muchos, por ejemplo: Peter Mc Laren, Michael Apple, Henry Giroux, Pérez Gómez y otros cuyas obras se difundieron en el país con posterioridad.

Las premisas señaladas se generaron lógicamente en un contexto pedagógico, propio de una escuela formadora de docentes, lo cual hace que estas no se queden en el discurso, sino que se canalicen hacia planes de estudio, o 
bien, que permeen diferentes ámbitos del discurso y de la práctica educativa; de ahí que su operacionalización tomara una vertiente pedagógica que implicaba múltiples elementos, como:

- $\quad$ Concepción de aprendizaje.

- Rol docente.

- Relaciones docente-estudiante.

- Relaciones estudiante-estudiante.

- $\quad$ Papel de los propósitos u objetivos en el quehacer pedagógico.

- Papel del contenido en ese quehacer.

- El rol de la evaluación y su conceptualización.

- $\quad$ El tipo de escuela idónea.

- El modelo curricular idóneo para las escuelas del país.

- Los espacios socioeducativos propicios para el inicio de un cambio educativo.

El concebir la educación como un elemento político y social, como una realidad ontológica con esas características, hará que en la práctica se vaya conformando una relectura y replanteamiento de los componentes básicos de un modelo pedagógico.

De esa concepción educativa, con claras connotaciones políticas, surgen dos preguntas fundamentales, las cuales en última instancia generaron consensos y disensiones, muchas de ellas, a veces, personalizadas por los actores sociales en el momento histórico que les toca actuar. En el caso que se analiza, dichas preguntas fueron las siguientes:

- ¿Qué tipo de sociedad se aspira construir con la acción educativa?

- ¿QQué tipo de seres humanos se desea intentar formar con la acción educativa?

Si bien es cierto estas preguntas no son nuevas, no implican ninguna revolución copernicana, son el eje de todo análisis filosófico en educación; el hacerlas explícitas lleva a grandes cuestionamientos, porque más allá del discurso, develan los sueños y las utopías de las y los formadores y de las y los estudiantes, así como de toda aquella persona que trate de mirar la acción educativa más allá de un mero instrumento de transmisión de saberes.

Las dimensiones y preguntas arriba expuestas, no implicaban, ni implican, un rápido consenso; tampoco su explicitación era y es importante para todos los académicos y académicas de un centro formador de docentes. 
Muchos docentes no tienen por prioridad establecer o problematizar en las relaciones entre educación-sociedad-política-sistema económico-sistemas de creencias, entre otros. No porque no los involucre, ya que de hecho eso involucra a todos, sino por múltiples razones que van desde la formación profesional hasta las experiencias históricas y cotidianas por las cuales han pasado, o bien, porque no se considera importante preguntarse sobre esos vínculos, ya que tales preguntas llevan a la polémica y al debate, prácticas a veces temidas sobre todo en sociedades como la costarricense.

Previo a la estructuración curricular de la Maestría en la DEB, se perfiló claramente una tendencia que podríamos denominar socioeducativa; esta tomó varias vertientes de concretización, entre los cuales se pueden citar tres:

a. Tendencia de discurso teórico conceptual.

b. Tendencia de acción pedagógica.

c. Tendencia dialéctica.

Si miramos estas tendencias a la luz del pensamiento de Paulo Freire (2004), encontramos su correlato en las siguientes categorías:

a. Discurso solamente reflexivo.

b. Discurso basado en la acción solamente práctica.

c. Discurso basado en la acción - reflexión - acción.

Freire enfatiza la atención que se debe poner en esta discusión, y es claro en señalar la tercera tendencia como la idónea en la formación docente; al respecto señala:

"La formación permanente de las educadoras, que implica la reflexión critica de la práctica, se apoya justamente en esta dialéctica entre la práctica y la teoría. Los grupos de formación en los que esa práctica de sumergirse en la práctica para iluminar en ella lo que en ella se da, y el proceso en el cual se da lo que se da, son, si se realizan bien, la mejor manera de vivir la formación permanente" (p. 124).

En la DEB, de los años noventa, las categorías freirianas no eran ajenas, así que dichas tendencias fueron asumidas por diferentes académicos y académicas. Se evita cualquier juicio, sobre el camino o ubicación en una u 
otra postura, obviamente que para Freire (2004), la tercera tendencia reviste el carácter ideal. Al diseñarse esta Maestría años después, la tendencia freiriana sería el centro de la propuesta pedagógica. De esa forma, la Maestría recoge la experiencia docente y la analiza a la luz del pensamiento crítico, la descompone y la resignifica mediante su problematización crítica.

Como se indicó, ante los cuestionamientos en torno al para qué social de la educación y sobre todo la idea de conceptualizar la Pedagogía como una acción política y social, llevó y lleva a la más profunda discusión y enfrentamientos académicos, porque quiérase o no, develan valores, ideales sociales, experiencias de vida, situaciones cotidianas; no obstante, es un ejercicio que se piensa necesario, si se pretende superar una visión instrumental de la educación. Pudiese pensarse que una instancia formadora de maestros y maestras que no posee esos enfrentamientos, esté pasando por un momento de armonía, pero esa armonía puede esconder el quehacer universitario de la reflexión y la confrontación de ideas, lo cual es paralizante y peligroso, para la conformación de un ambiente crítico y propositivo. De los enfrentamientos pedagógicos abiertos y fuertes que vivió la $\mathrm{DEB}$, en los años noventa, la Maestría en Pedagogía recogió los frutos de una batalla académica y conceptual.

Al iniciarse un debate en tan amplio espectro como lo son las implicaciones políticas y sociales de la educación, la DEB generó debate en diversas áreas, y el cuestionar el ahora llamado modelo pedagógico, implicó revisar los elementos arriba mencionados; muchas veces, por ejemplo, al debatir en torno a la concepción de aprendizaje, a la concepción de distribución del espacio en el aula, se entra en la discusión del referente social, aunque no se tuviese una conciencia explícita de ello.

En la década de los noventa, la DEB tuvo esa constante discusión sobre los componentes de un modelo pedagógico. Es claro a la luz del tiempo, analizar la posición que se fue fraguando en la $\mathrm{DEB}$, posición que en la mayoría de los componentes citados no lograba un empate con lo propuesto y sobre todo con la operacionalización del modelo pedagógico propuesto por el Ministerio de Educación Pública. Lo anterior, sobre todo en las preguntas clave del tipo de sociedad que el modelo deseaba impulsar.

La DEB optaba por un modelo con características profundamente críticas, que, en su médula, cuestionaba el sistema social, económico político, vigente. Por eso, y en ese sentido, la Maestría en Pedagogía con énfasis en Diversidad en los Procesos Educativos nace desde el discurso de la contestación, de la crítica y no es complaciente con modelos homegeneizantes y 
normativos de educación, hereda pues la relación dicotómica y crítica de la DEB de los noventa con respecto a aquellas propuestas ministeriales que buscan homogeneizar, estandarizar los procesos educativos y a los individuos inmersos en ellos.

Sin embargo, es importante señalar que esa posición de la $\mathrm{DEB}$ en los años noventa, implicó un proceso de contradicción y disenso, ya que no todos los actores sociopedagógicos, y sobre todo los académicos, compartían el ideal más alto del modelo pedagógico, la transformación social. Para muchos, implicaba una posición radical, extrema, que no reflejaba los compromisos ideológicos de muchas personas. No obstante, la tendencia con mayor o menor fuerza, se mantuvo y mantiene hasta la actualidad y será uno de los pilares fundamentales de la creación de la Maestría en Pedagogía con énfasis en diversidad en los procesos educativos. Es necesario señalar que dicha tendencia encontró un espacio de desarrollo sobre todo en la carrera de I y II ciclos; esto no desmerita los debates y creaciones discursivas que se generaron al interior de otras carreras propias de la División; pero estas no tuvieron un peso determinante en la creación de la Maestría en Pedagogía con énfasis en Diversidad en los Procesos Educativos.

\section{Relación entre demandas de la realidad socioeducativa nacional y la Maestría en Pedagogía}

El ocaso de los noventa y la alborada del nuevo siglo, abren la puerta a un nuevo proceso, que tímidamente se gestaba en ámbitos internacionales, la legalización de los procesos de integración de personas con discapacidad a los procesos educativos considerados como "homogéneos" y "normales", y de paso la ruptura total con la concepción segregacionista de personas con discapacidad en el sistema educativo nacional. La Ley 7600 ilumina el debate en la DEB en la carrera de Educación Especial. No tan vinculado con el discurso de la contradicción de las clases sociales. La carrera de Educación Especial encuentra o explicita, poco a poco, que sus principales beneficiarios, personas con discapacidad, son un sector excluido del sistema educativo formal, cuyos más elementales derechos son obviados, por un sistema educativo homogeneizante y excluyente. Es decir, la categoría de excluido cobra una amplitud en el pensamiento de la DEB, superando el sello de exclusión por clase social, y ampliándose al de exclusión por discapacidad. Esta concepción se ve claramente presente en el plan de estudios de la Maestría, cuando se indica lo siguiente: 
"Cabe señalar que se entiende la diversidad como aquella que responde no sólo a grupos culturales o étnicos sino también de género, clase social, estilos y formas particulares de aprender, y personas con discapacidad o sobrecapacitadas" (p. 27).

A la luz del tiempo, el hecho de la promulgación de la Ley 7600, y el compromiso asumido por la carrera de Educación Especial de la DEB, fueron fundamentales en la creación de la Maestría en Pedagogía, ya que implica una ruptura en la cosmovisión de los académicos de la DEB; la integración devela que el sistema educativo es excluyente y está al servicio de la "normalidad", que es injusto y poco equitativo.

La contradicción con el sistema educativo formal, vivida y promulgada por un grupo de académicos de la $\mathrm{DEB}$, sobre todo vinculados con la carrera de I y II ciclos, centrada en el componente socioeconómico, encuentra un aliado en un grupo importante de la carrera de Educación Especial, la cual clarifica su contradicción mediante las resistencias de muchos a creer en la posibilidad de la integración de las personas con discapacidad al sistema educativo formal. La exclusión de seres humanos, pasa a ser un punto de convergencia ideológica entre dos carreras que compartían un espacio físico, pero no un ideal social. La premisa se modifica; se converge en la existencia de un sistema social excluyente tanto de niñas y niños pobres como de personas con discapacidad. La discusión de la DEB encuentra, además, que el pilar ideológico que sustenta las desigualdades, es el sistema educativo formal. Lo anterior, profundiza la necesidad de una respuesta pedagógica que tienda a no ser convergente con lo propuesto por el Ministerio de Educación Pública en sus más profundos ideales, elementos coyunturales y discusiones compartidas son posibles, siempre y cuando, no se profundice en la utopía social de uno u otro actor.

Esta contradicción entre centros universitarios formadores de docentes y propuestas gubernamentales oficiales, es una de las importantísimas excepciones de Costa Rica en el contexto centroamericano; la universidad no puede y no debe estar supeditada a la sumisión de la política de los ministerios; puede presentar alianzas coyunturales, pero el papel de la universidad es ser la conciencia crítica de la sociedad y, por lo tanto, la conciencia crítica de las propuestas oficiales y gubernamentales, la educación no es la excepción. Lo anterior no implica una confrontación estéril, ni una separación absurda; pero la naturaleza social de la universidad pública costarricense, hace que siempre las propuestas estatales (entendiendo el Estado como un elemento 
de negociación social) sean objeto de reflexión y crítica académica; en las universidades, las posiciones acríticas no tienen cabida.

Otros cambios en la dinámica mundial, hacen que el pensamiento de la $\mathrm{DEB}$ en torno al quehacer del ideal educativo sufra una serie de replanteamientos, los cuales permitirán ampliar el espectro de acción y la conformación del discurso que el posgrado asumirá, totalmente vinculado con una educación para diversidad en un sentido amplio. De esa forma, el fin del socialismo real, concretizado en las naciones del este de Europa, genera una crisis en el pensamiento político mundial, factores como los nacionalismos, la xenofobia, la homogeneización de la preferencia sexual y el feminismo, son temas que aparecen ahora como luchas por la equidad social, política, económica. Los debates mundiales y nacionales abren puertas que antes aparecían ocultas por los conflictos eminentemente socioeconómicos.

En el caso del desarrollo del pensamiento de la División de Educación Básica, uno de los principales temas emergentes de discusión lo constituyó y constituye la inmigración de familias nicaragüenses al país y, sobre todo, la presencia de niñas y niños de esa nacionalidad en las aulas regulares del sistema educativo nacional. De esa forma, autores como los lejanos Bowles y Gentis (1975), o los primeros escritos de Peter Mc Laren (1981) que develaban la exclusión de que eran víctimas minorías étnicas en los Estados Unidos, encuentran un correlato directo y visibilizado en el quehacer pedagógico del país y en la discusión de los académicos y las académicas de la DEB. La creciente migración de nicaragüenses empieza a generar un reflejo directo y una nueva pregunta para la formación docente en la DEB. Los procesos formales de educación y la práctica de muchas escuelas, no solo excluían por clase social y discapacidad, sino que ahora también lo hacían por nacionalidad y etnia.

La formación docente en los programas de pregrado y grado no abarcaba estos nuevos escenarios, no los contemplaba, no por miopía o ceguera conceptual, sino porque estos nuevos retos surgieron aceleradamente, cosa que no es de extrañar. No obstante, es en el diseño curricular de la Maestría en Pedagogía con énfasis en Diversidad en los Procesos Educativos, que las demandas sociales antes mencionadas encuentran una primera respuesta por parte de la DEB. Es importante insistir en el hecho de que la dinámica social tiende a rebasar las respuestas institucionalizadas y aún más las respuestas curriculares, las cuales, si se consideran serias, requieren de estudios, análisis rigurosos y consensos entre expertos académicos. Construir un proyecto curricular de calidad no implica recortar y pegar. Diseñar currículo es crear 
un proyecto social, una hipótesis de desarrollo; como lo señala Posner (2000, p. 81), las propuestas curriculares de calidad deben tener claros los niveles y propósitos educativos, estos suelen incluir diversos tipos de metas, tales como: las de la sociedad, las administrativas, los fines educacionales, las metas educacionales y los objetivos de aprendizajes (objetivos entendidos como lo que se espera de la persona cuando finaliza un programa curricular). No es de extrañar que la respuesta de la DEB no fuera inmediata, sino que se concretizara en la Maestría a inicios del nuevo milenio.

Otras tendencias de menor impacto aparecieron en el ámbito de la DEB: la presencia de exclusión por preferencia sexual y la exclusión por la condición de género.

La primera de ellas surge más que todo, por el intento en la División de Educación Básica de superar los esquemas positivistas de investigación en educación. La búsqueda de experiencias investigativas profundamente cualitativas, produce el encuentro con los trabajos de esa naturaleza realizados por Jacobo Shifter Sikora, entre los que se pueden citar los siguientes: Amor de Machos, Lo que la abuela nunca nos contó sobre las cárceles (1998), Caperucita Rosa y el Lobo Feroz, Sexo Público Latino (1999) y De Ranas a Princesas. Sufridas, atrevidas y travestidas (1998).

Los textos de Shifter no llegan al seno de la DEB, y sobre todo a la carrera de I y II ciclos, en forma masiva, sino que el camino es a través de los cursos de investigación y su presencia es ante todo metodológica más que temática. No obstante, fondo y forma son caras de una misma moneda, sobre todo en el enfoque filosófico de la totalidad. Las obras de Shifter consultadas apuntan a la presencia de actores marginales y excluidos no por clase social, discapacidad o etnia, sino por preferencia sexual: el travesti, el prostituto, el gay en diferentes espacios sociales, encuentran una presencia en el discurso pedagógico de la DEB a través de lo metodológico, pero de una u otra forma se visibiliza su existencia y termina convirtiéndose, tímidamente, en un reto educativo por atender.

El caso de la exclusión por género no logra una impronta fuerte en la División de Educación Básica, los ecos de dicha contradicción provienen más que nada de otras unidades académicas, sobre todo de la División de Educología; igual sucede con la visibilización de las poblaciones indígenas, cuyo clamor como etnia excluida llega a la DEB, proveniente de la División de Educación Rural.

Otros retos de menor fuerza aparecen mediante las voces de las y los estudiantes de los cursos de práctica y docentes en servicio que conti- 
núan estudios dentro de la $\mathrm{DEB}$, como lo son la presencia de niñas y niños con extraedad en los grupos, las madres jefas de hogar y el abuso infantil, la atención a niños y niñas con nuevas visiones de mundo, con procesos de desarrollo humano acelerados con respecto a la teoría y a la práctica nacional. Esas preguntas e inquietudes estudiantiles son retos que obligan a la formulación de un currículo diferente. El cauce o canalización de los retos (en forma conjunta) será la Maestría en Pedagogía con énfasis en Diversidad en los procesos educativos.

\section{Logros y alcances de la Maestría}

En el período administrativo 1995-2000, las autoridades de la DEB señalan como una de sus acciones prioritarias, la creación de un posgrado, y realizan un intenso trabajo para diseñar una propuesta de Maestría en Didáctica; por razones que no son pertinentes para este artículo, la maestría en mención no se ejecutó.

La siguiente administración 2000-2005, decidió retomar tal línea de acción, con una inteligente conformación del equipo de diseño curricular, ya que el mismo fue estructurado por académicos y académicas pertenecientes a las carreras de I y II ciclos de la Educación General Básica y Educación Especial. En ese momento, no era fácil mirar los bagajes ideológicos que respaldaban a las carreras citadas tal y como se ha hecho en este artículo; pero ambas tenían como ideal educativo superar los procesos de exclusión mediante la formación de docentes con una visión crítica de la pedagogía, que tuvieran la capacidad de elaborar propuestas curriculares, didácticas ya fuera para centros educativos o para aulas, que fuesen alternativas a las propuestas nacionales oficiales consideradas funcionales a diferentes formas de exclusión.

La convergencia en las mestas educacionales, en el ideal de profesional y de ser humano que se perseguía, hizo del trabajo de dicha comisión curricular un proceso ágil y posible. Si bien es cierto, ningún proceso de díseño curricular es fácil, cuando existen idearios compartidos en las metas educacionales, los procesos tienden a ser más ágiles. La comisión estructuró la Maestría en el lapso de un año. Para el año 2003, se abrió la primera promoción, acción que se repitió en los años 2004, 2005 y 2006.

No es el propósito del artículo reseñar el desarrollo de la Maestría per se, bien se mencionó al inicio que el objetivo fundamental lo es mirar la conformación de un plan de estudios como producto de la convergencia de 
procesos micro y macrosociales; solamente y a modo de cierre, interesa mostrar que el ideal educativo ha rendido sus frutos académicos; de esa forma, la primera promoción desarrolló sus trabajos finales de investigación sobre las siguientes temáticas:

1. Evaluación del Programa Aula Abierta en espacios urbano-marginales.

2. Habilidades para la vida en niños institucionalizados del Patronato $\mathrm{Na}$ cional de la Infancia y un centro educativo privado.

3. Propuesta para la atención a los padres de familia de niños y niñas con síndrome de Down.

4. La incorporación de la diversidad cultural en textos de IV grado.

5. Propuesta de desarrollo profesional a docentes que trabajan en escuelas con población migrante nicaragüense.

6. Propuesta de formación en atención a la diversidad para docentes que laboran en escuelas urbano-marginales.

7. Propuesta de formación en atención a la diversidad a niños y niñas de I grado en escuelas regulares de I y II ciclos.

8. Incorporación de la atención a la diversidad en los procesos de lectura y escritura.

9. Propuesta en atención a la diversidad para docentes y personal que labora en los CEN-CINAI.

10. Propuesta de desarrollo profesional en atención a la diversidad dirigido a docentes que laboran en centros educativos de I y II ciclos.

La segunda promoción elaboró sus trabajos finales de graduación en líneas muy similares, y de igual forma apuntan los trabajos de la tercera promoción.

Como se mencionó, el ideario pedagógico de la DEB encuentra su punto convergente en el análisis de los procesos de exclusión mediante las acciones pedagógicas, así como en la construcción y análisis de las respuestas que, de una u otra forma, tratan de eliminar tales procesos de exclusión; los trabajos que se presentan en esta Revista son muestras de ello y los primeros frutos de una maestría que nace contestataria, propositiva y subversiva, privilegios que otorga la universidad pública costarricense. De esa forma, en los siguientes artículos, se podrá mirar la operacionalización de muchas de las ideas aquí expuestas, y de paso se logrará notar que los más altos ideales del diseño curricular de la Maestría cobran vida propia en las propuestas de trabajos finales de la primera promoción de la Maestría en Pedagogía con énfasis en Diversidad en los Procesos Educativos. 
Finalmente, es importante señalar que los procesos de diseño curricular, responden a profundos sueños, utopías, consensos y discensos sobre imaginarios sociales; el éxito no radica en lograr grandes cantidades de dinero, sino en formar docentes que no permitan ningún tipo de exclusión e inequidad. El diseño curricular implica momentos de convergencia entre macroprocesos y procesos microinstitucionales, de los cuales los actores sociales no siempre son conscientes del todo. La dinámica curricular posee una perspectiva histórica, que nunca se debe soslayar. 


\section{REFERENCIAS}

Ayuste, A., Flecha, R., López, F. y Lleras J. (1998). Planteamientos de la Pedagogía Crítica. Barcelona, España: GRAO.

Florez, R. y Tobón, A. (2001). Investigación Educativa y Pedagogía. Bogotá, Colombia: Mc Graw Hill.

Bowles, S. y Gintis, H. (1981). La instrucción escolar en la América Capitalista. Madrid, España: Siglo XXI Editores.

Freire, P. (2004). Cartas A Quien pretende Enseñar: Buenos Aires, Argentina: Siglo XXI Editores.

Mc Laren, P. (2003). Pedagogía, Identidad y Poder: Los Educadores frente al Multiculturalismo. Rosario, Argentina: Homo Sapiens Ediciones.

Posner, G. (1998). Análisis del Currículo. Bogotá, Colombia: Mc Graw Hill.

Ritzer, G. (2000). Teoría Sociológica Moderna. Madrid, España: Mc Graw Hill.

Shifter, J. (1998) Amor de Machos. Lo que la abuela nunca nos contó sobre las cárceles. San José, Costa Rica: ILPES.

Shifter, J. (1999). Caperucita Rosa y el Lobo Feroz. Sexo Público Latino. San José, Costa Rica: ILPES.

Shifter, J. (1998). De Ranas a Princesas. Sufridas, atrevidas y travestidas. San José, Costa Rica: ILPES.

Torres, J. (2001). Educación en tiempos de neoliberalismo. Madrid, España: Morata Editores. 